\title{
Impact of exogenous progesterone on ovarian follicular dynamics and function in mice
}

\author{
E. Telfer ${ }^{1, *}$, R. G. Gosden ${ }^{1, \dagger}$ and M. J. Faddy ${ }^{2} \ddagger$ \\ ${ }^{1}$ Department of Physiology, University Medical School, Edinburgh EH8 9AG, UK; and \\ ${ }^{2}$ Department of Statistics, University of Birmingham B15 2TT, UK
}

\begin{abstract}
Summary. Raising progesterone concentrations in young adult mice by subcutaneous implants resulted in ovulation being blocked and the cessation of oestrous cycles. The effect of this treatment on the numbers and dynamics of preantral follicles during 36 days of treatment was studied using a compartmental model to analyse differential follicle counts. Changes in growth and/or death rates were detected at all stages of follicular development. An increased rate of growth through preantral stages was predicted in the treatment group when compared with the controls, but most of these follicles did not reach the antral stage of development as an increased death rate was observed at large preantral stages (stage IV). Antral follicles were formed in the treatment group, but all succumbed to atresia. Increased atresia in the antral population of follicles in the treatment group was observed directly.
\end{abstract}

Keywords: progesterone; mathematical modelling; ovarian follicles; mouse

\section{Introduction}

The suppression of ovulation when conditions for conception are physiologically unfavourable, as occurs, for example, during pregnancy, lactation and seasonal food/water shortages, is presumably advantageous, since it is almost universal in mammals. However, the causes of ovulation inhibition and its effects on follicular growth and death are far from understood, despite extensive study.

Anovulation during pregnancy is generally thought to depend on diminished pulsatile secretion of gonadotrophic hormones, follicle-stimulating hormone (FSH) and luteinizing hormone (LH), which has been demonstrated in humans and in some animals (Linkie \& Niswender, 1972; Murr et al., 1974; Baird et al., 1984; Jeffcoate, 1986). Consequently, follicles are not expected to complete their maturation to Graafian stages (Richards, 1980). In some species, Graafian follicles are absent during most of gestation whereas in others they may form, but undergo atresia rather than ovulate. Much attention has been paid to large follicles, but there is little information about the impact of anovulation on the dynamics of preantral follicles (Pedersen \& Peters, 1971). Since these follicles constitute a reserve which limits the capacity for ovulation and the potential length of functional ovarian life, the dynamics of the preantral stages should be clarified during pregnancy and in other states in which ovulation is inhibited.

The present study estimated the rates of follicle growth and death in mice given progesterone implants to induce a state of anovulation. This approach was adopted because it was likely to produce a steady-state condition which would facilitate analysis, as opposed to the complex and changing physiological conditions of pregnancy and pseudopregnancy. The dynamics of preantral

*Present address: The Jackson Laboratory, Bar Harbor, Maine 04609, USA.

$\dagger$ Requests for reprints.

$¥$ Present address: Department of Mathematics and Statistics, University of Otago, PO Box 56, Dunedin, New Zealand. 
follicle growth and death were investigated using differential follicle counts, analysed using an established mathematical model (Faddy et al., 1976, 1987).

\section{Materials and Methods}

General procedures. Oestrous cycles of virgin female CBA/Ca mice were monitored by examining vaginal smears 6 days per week from $40-45$ days of age until the mice were killed. Only those presenting three or more consecutive 4-or 5-day cycles at the beginning of the study were used.

Hormonal implants were made from $1 \mathrm{~cm}$ lengths of sterile Silastic tubing (Dow Corning, USA) with an internal diameter of $1.9 \mathrm{~mm}$. They were packed with crystalline progesterone (Sigma, London, UK) and sealed with silicone adhesive (Dow Corning, USA). The implants were incubated overnight in sterile saline at $37^{\circ} \mathrm{C}$ before being inserted subcutaneously in the nape region during tri-bromoethanol anaesthesia $(0.63 / \mathrm{kg}$, i.p. body weight). Since the effective life of the implants was $\sim 35-40$ days, they were replaced every 30 days under brief anaesthesia.

The efficacy of treatment was tested in 24 animals which were divided randomly into three groups receiving at pro-oestrus either an empty implant or one or two hormonal implants. Blood specimens were obtained under anaesthesia by cardiac puncture after 16 days of treatment and the animals were killed by cervical dislocation before regaining consciousness. The ovaries and oviducts were dissected and examined microscopically $(\times 25)$ for corpora lutea, follicle stigmata and ovulated oocytes. The progesterone concentrations in the sera were analysed by radioimmunoassay of which the characteristics have been described elsewhere (Fraser, 1983). On the basis of preliminary studies, one hormonal implant was shown to be sufficient to block ovulation for up to 30 days and was therefore chosen for subsequent experiments.

Numbers and dynamics of follicles. Thirty-five animals were given either a control implant $(n=6)$ or a hormonal implant $(n=29)$ at 55 days of age. Vaginal smears were obtained daily until autopsy, which ranged from 5 to 36 days later and occurred on the morning of pro-oestrus in control animals. Another group of animals $(n=11)$ was treated from the same ages with implants for up to 120 days along with seven control animals.

Implants were examined at autopsy to confirm that crystalline hormone remained. The ovaries were removed, fixed in aqueous Bouin's fluid and prepared as $7 \mu \mathrm{m}$ serial sections stained with haematoxylin and eosin. Follicles with up to four granulosa cell layers were counted in every tenth section using the nucleolus of the oocyte as a marker; larger follicles were traced through every section. The total numbers of follicles per animal were estimated and assigned to stages of development (I-IV) using the classification of Mandl \& Zuckerman (1951). Large follicles with an enlarged extracellular space were subclassified according to whether the antrum was incipient (VIa) or had formed (VIb). Follicles at stages V and VI were considered 'atretic' if they contained three or more pyknotic granulosa cells in the equatorial section.

The numbers of healthy and atretic follicles were compared in control and experimental animals at different ages. Since the data from animals with control implants and those from a detailed concurrent study of intact animals were similar (Faddy et al., 1987), the latter set of data and the model derived from it were used as the primary bases for comparisons. The dynamics of follicle growth and death in preantral stages were estimated for the age range 55-91 days using a compartmental model (Faddy et al., 1976) and nonparametric regression analysis guided the fitting of the model (Faddy \& Jones, 1988). The Kruskal-Wallis analysis of variance of ranks was used to compare numbers of large follicles in treated and control animals.

\section{Results}

\section{Efficacy of hormonal implants}

Normal vaginal smear patterns stopped abruptly in the hormonal implant group whereas animals in the control group continued to present normal oestrous cycles. In the former group, smears became persistently dioestrous within 2 days of treatment and oviducal oocytes and hyperaemic corpora lutea were absent at post mortem. The animals appeared to remain healthy throughout the study. Plasma progesterone concentrations were $22 \pm 4 \mathrm{ng} / \mathrm{ml}$ (mean \pm s.e.m.), these being significantly greater than controls, $10 \pm 3 \mathrm{ng} / \mathrm{ml}(P<0.001)$.

\section{Numbers and dynamics of follicles}

For animals aged 55-91 days, there were fewer (27-61\%) follicles at stages II, III and IV in progesterone-treated animals than in normal controls (Fig. 1), whereas the numbers of stages I$\mathrm{V}+$ (stages V, VIa and VIb were combined into a single stage $\mathrm{V}+$, as in Faddy et al., 1987) were similar. These data therefore suggested that an existing model for normal animals is inadequate for describing the conditions of progesterone domination and anovulation (Faddy et al., 1987). A new 

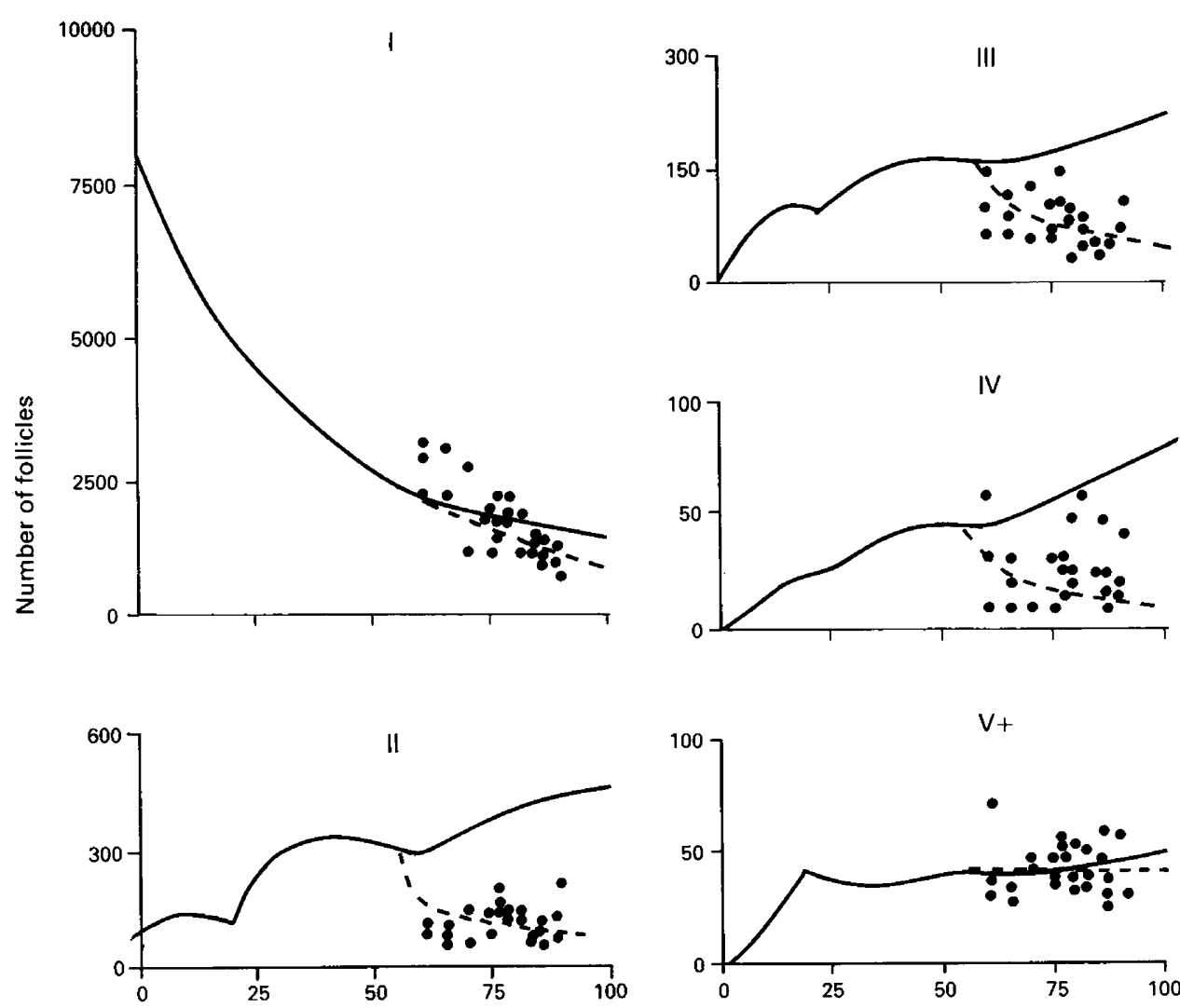

Age (days)

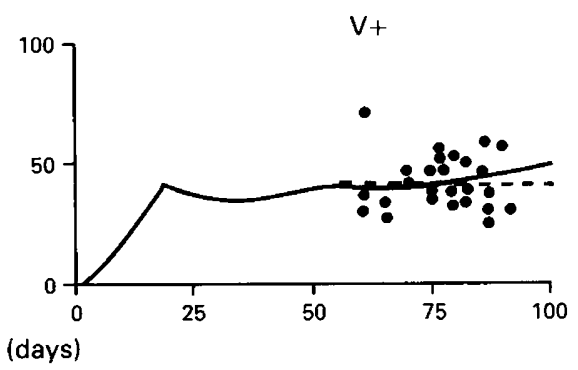

Fig. 1. Numbers of follicles at five stages of growth $(\mathrm{I}-\mathrm{V}+$, with $\mathrm{V}+$ being a combination of $\mathrm{V}$, $\mathrm{VIa}$ and VIb) in CBA/Ca mice which received, at 55 days old, an implant containing progesterone to inhibit ovulation. The compartmental model (-- ) has been fitted to these data (see also Fig. 2), and the solid curves ( - ) describe age changes in the ovaries of normal animals.

compartmental model was therefore fitted: this model compared well with the nonparametric regressions of the data - the sum of squares for the data relative to the fitted model being only $7 \%$ greater than the sum of squares for the nonparametric regressions (Table 1).

Table 1. Average numbers of follicles entering and leaving successive stages of development (per day) in progesterone-treated mice, as estimated from a compartmental model (see Fig. 2)

\begin{tabular}{lccccccccc}
\hline Age & & & \multicolumn{7}{c}{ Stage of development } \\
(days) & $\mathrm{I} \rightarrow$ & $\rightarrow \mathrm{II}$ & $\mathrm{II} \rightarrow$ & $\rightarrow \mathrm{III}$ & $\mathrm{III} \rightarrow$ & $\rightarrow \mathrm{IV}$ & $\mathrm{IV} \rightarrow$ & $\rightarrow \mathrm{V}+$ & $\mathrm{V}+\rightarrow$ \\
\hline 63 & 35 & 35 & 42 & 42 & 48 & 48 & 50 & $0 \cdot 3$ & $0 \cdot 1$ \\
70 & 31 & 31 & 34 & 34 & 36 & 36 & 37 & $0 \cdot 2$ & $0 \cdot 1$ \\
77 & 28 & 28 & 30 & 30 & 31 & 31 & 32 & $0 \cdot 2$ & $0 \cdot 2$ \\
84 & 25 & 25 & 27 & 27 & 28 & 28 & 25 & $0 \cdot 2$ & $0 \cdot 2$ \\
91 & 22 & 22 & 24 & 24 & 25 & 25 & 22 & $\mathbf{0} \cdot 2$ & $\mathbf{0 \cdot 2}$ \\
\hline
\end{tabular}

The estimated growth and death rates for the model are shown in Fig. 2, and the resulting fit to the data is demonstrated in Fig. 1, which also shows the curves derived from modelling data obtained from normal CBA/Ca animals (Faddy et al., 1987). It is clear that these results for 
progesterone-treated animals differ substantially from those for normal animals. These differences could not be explained in terms of changed death rates alone, since fitting a model constrained to normal growth rates (Faddy et al., 1987) resulted in a significantly $(P<0.01)$ poorer fit, and high estimated death rates at stages I-III which did not accord with morphological observations. There was no evidence of any age dependence in the growth and death rates during the study.

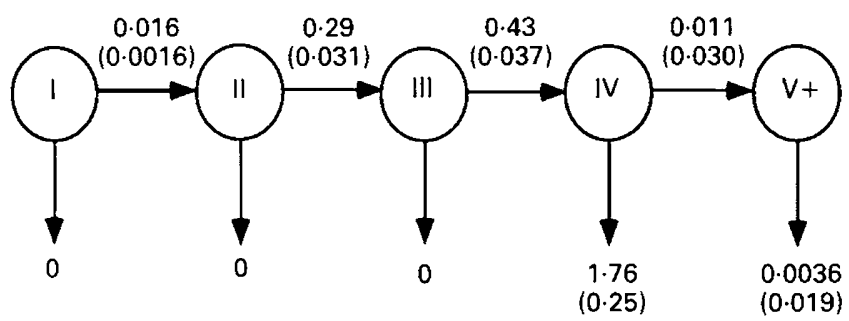

Fig. 2. Estimated mean rates of follicle growth and death per day, with s.e. in parentheses, for each of the five successive stages ( $\mathrm{I}$ to $\mathrm{V}+$ ) in young adult mice, 55-91 days old, treated with progesterone.

Similarities as well as differences were observed in the models for control and treatment groups. In both cases, the death rates among small follicles were negligible, but the rate of growth from stage I was significantly greater in treated animals $(0.06 \mathrm{cf} .0 .0051, P<0.001)$. The rates of movement between stages II and IV were appreciably greater in these animals $(P<0.001)$, as indicated by the abrupt fall in numbers. Since follicle development in anovulatory ovaries is not suspended, growing follicles die at some stage. Most deaths occurred at larger stages, particularly stage IV, which checks an otherwise large inflow into the antral population and a consequent expansion of ovarian mass. The rate of recruitment into stage $\mathrm{V}+$ is reduced by a factor of ten from an average of $\sim 2$ per day in normal animals. Table 1 shows the average numbers of follicles leaving and entering successive stages, for comparison with those for normal animals (Faddy $e t$ al., 1987).

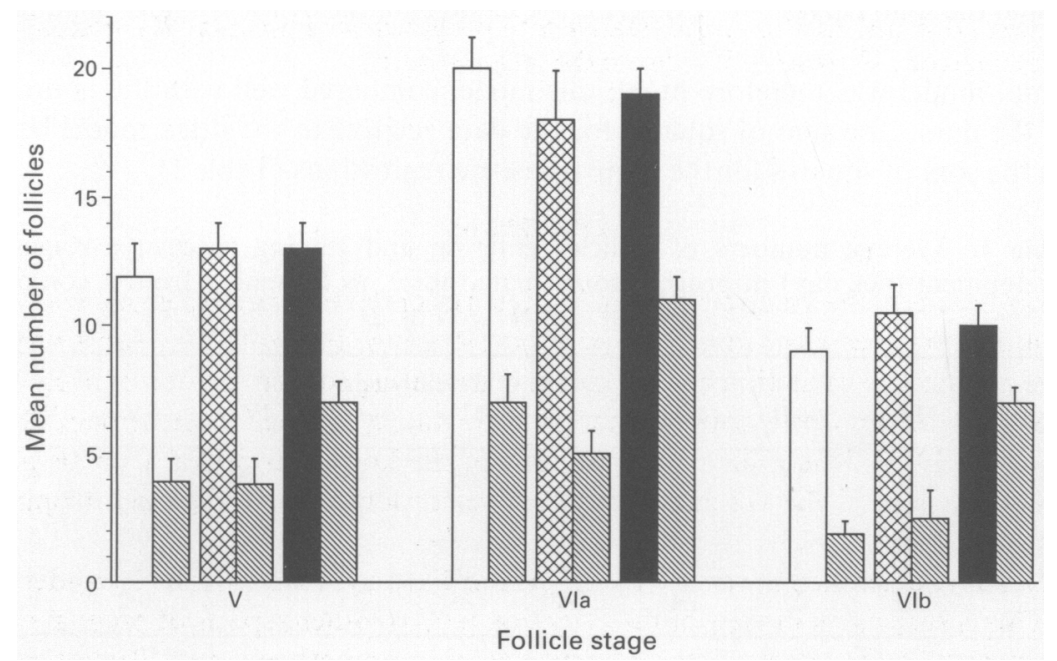

Fig. 3. Numbers of follicles at three stages of development in normal mice ( $\square$; from data from Faddy et al., 1987) at pro-oestrus and in progesterone-treated ( $\boldsymbol{\square})$, anovulatory mice, 60-100 days old and in sham controls ( The follicles are classified on the basis of their histological appearance into healthy and atretic $(\mathbb{\mathbb { Q }})$ subgroups. Vertical bars indicate the mean \pm s.e.m. 
The generally comparable numbers of stage $\mathrm{V}+$ follicles, as a whole, obscures complex differences within this heterogeneous group. The model cannot provide precise estimates of the dynamics at this stage (Fig. 2) and so inferences have been drawn directly from morphological evidence. Figure 3 shows the mean number of healthy and atretic follicles at stages V, VIa and VIb in three groups of animals aged 60-100 days. The normal controls refer to those animals from a concurrent study published earlier (Faddy et al., 1987) and are shown here to demonstrate that the results for shamoperated controls and the other controls are virtually identical. The mean number of large follicles in each of stages $\mathrm{V}$, VIa and VIb of antral development were not significantly different in control and treated animals (Fig. 3), but the number of atretic follicles at each stage was significantly greater in the progesterone-treated group $(P<0.0025)$. These differences can be illustrated by the mean percentages of atretic follicles, which clearly showed an excess of atresia in the treatment group; in some cases the differences were more than twofold (Fig. 4).

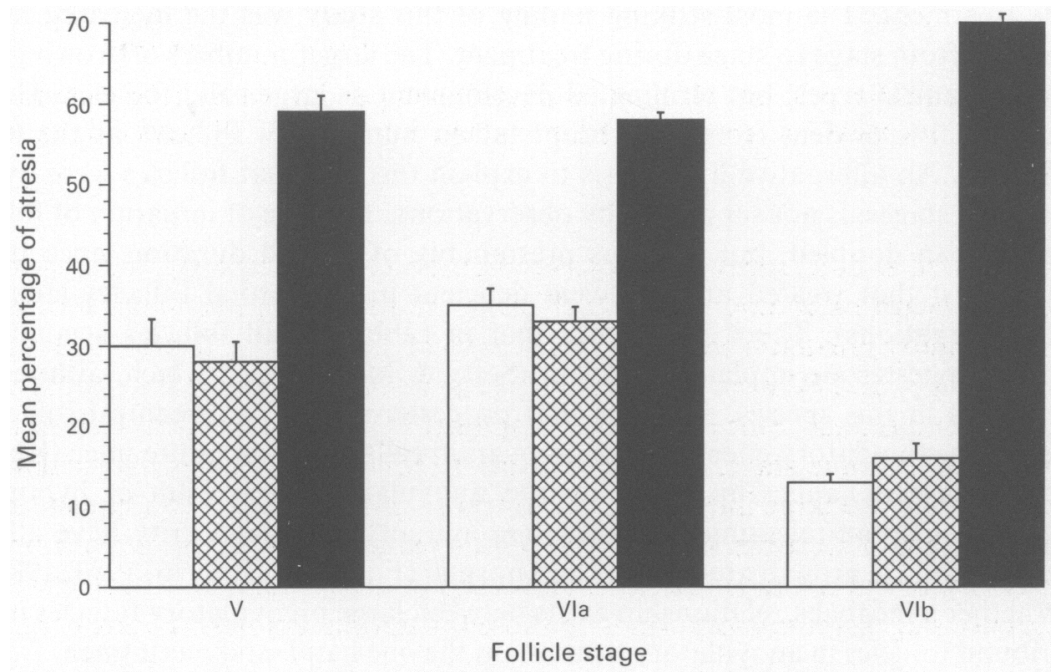

Fig. 4. Percentages of follicles at three stages of development that were atretic in pro-oestrous cyclic mice and in anovulatory mice treated with progesterone implants $(\boldsymbol{\square})$; normal $(\square)$ and sham () controls. Vertical bars indicate the mean \pm s.e.m.

\section{Discussion}

This study was based on the assumption that implants of crystalline progesterone maintain a steady ovulatory state, as has been known for many years (Robson, 1938). The implants of progesterone were effective at blocking ovulation and producing circulating concentrations of progesterone comparable with those of early pseudopregnancy and pregnancy in mice (McCormack \& Greenwald, 1974; Murr et al., 1974). The follicular physiology in the hormone-treated animals is expected to differ, however, because of the absence of both active corpora lutea and gonadotrophic hormones from the placenta/decidua.

Few studies have examined the behaviour of primordial and preantral follicles under anovulatory conditions. This is surprising in view of the attention paid to other aspects of pregnancy physiology and general interest in the effect of steroid contraception on pathogenesis. Studies carried out on pregnant rats have shown that only small preantral follicles are present throughout pregnancy and pseudopregnancy; only at the end of pregnancy do large follicles emerge (Greenwald, 1966). Evidence of inhibitory effects of exogenous progesterone on follicular development has been 
obtained in a number of species, e.g. rats (Buffler \& Roser, 1974), hamsters (Greenwald, 1977) and rhesus monkey (Goodman \& Hodgen, 1977); progesterone may have direct effects on follicular development (Moore \& Greenwald, 1974; Schrieber et al., 1980), but it does not necessarily follow that this is the hormone responsible for effects on follicles during pregnancy.

Some studies have looked at antral follicles during pregnancy (Greenwald \& Choudary, 1969), but only one investigation of follicle dynamics in pregnant mice has been carried out and this used the labelled mitosis technique (Pedersen \& Peters, 1971). The results showed that follicles grew at similar rates to those in non-pregnant animals, but the rate of entry of follicles from the primordial pool to the growing stages was reduced. To what extent the differences between this result and the present study can be attributed to the different physiological conditions remains conjectural.

The model obtained from the present study gives a holistic impression of the dynamics of follicles since it encompasses both follicle growth and death. The compartmental model shows that the growth and death rates at all stages of follicular development have been affected by progesterone treatment. The most striking finding of this study was the increased movement of preantral follicles from stage to stage during treatment. The larger numbers of growing follicles did not all emerge as antral types, but terminated development as large solid follicles. This interpretation by the model is evident from the abrupt fall in numbers of follicles at the beginning of treatment (Fig. 1). An alternative hypothesis to explain this fall, that follicles were dying in large numbers at earlier stages, is not supported by observations. The rate of initiation of follicle growth (i.e. I-II) more than doubled, but this was presumably of limited duration since there was no evidence to suggest that treated animals were deficient in primordial follicles later (E. Telfer, unpublished observations). There is evidence that in rabbits small follicles accumulate during pregnancy and progesterone implant treatment (Setty \& Mills, 1987), which, although implying different dynamics in this species, confirms that progesterone affects preantral follicles. Indeed, progesterone has a stimulatory effect in vitro on ovarian cells from immature mice (Kent, 1973).

Studies of follicle dynamics in mouse ovaries anovulatory as a result of hypophysectomy, absence of gonadotrophin-releasing hormone ( $p g$ mutant) and immaturity have all found that rates of preantral follicle growth are greater than normal (Faddy et al., 1976, 1983, 1987; Halpin et al., 1986). Whether a feedback relationship exists between large preovulatory follicles in cyclic mice or large moribund follicles in anovulatory ovaries, on the one hand, and recruitment from the small follicle reserve, on the other, is a hypothesis which warrants experimental testing. Since the pulsatile release of pituitary FSH concentrations is reduced in pregnancy as a result of inhibition by steroid hormones, it would appear unlikely that this is the effector of accelerated growth of small follicles. The existence of hypothetical feedback would ensure continuing replenishment of follicle recruits such that rapid resumption of ovulation could occur when inhibitory influences, such as lactation or pregnancy, end.

In contrast to the rapid movement of small follicles, the model predicts an increased death rate of stage IV follicles, which counteracts the increased growth of earlier stages. Although ovulation was blocked, follicles still proceeded to form an antrum, but the rate was considerably more sluggish. Clearly, there is a problem when comparing numbers of large follicles in anovulatory animals and others having cyclic variation, but comparisons made for preantral follicles need have no regard for this factor (Numazawa \& Kawashima, 1982). All large follicles in progesteronetreated animals succumbed to atresia. The incidence of atresia as determined by morphological criteria was greater in the treatment group and is probably a result of low concentrations of gonadotrophins. Persistence and accumulation of atretic follicles in pregnant and pseudopregnant rats (Osman, 1985, 1986) might explain the substantial increase in atresia noted at stage $\mathrm{V}+$ in progesterone-treated mice.

This study found effects of suppression of ovulation by progesterone on all stages of follicular development, including primordial. Further study is required to determine at which stages, if any, these effects are direct. It is unlikely that progesterone acts directly on the primordial population to increase the initiation of growth, but this increase may be related to the effects on the large follicles. 
An alternative approach will have to be taken and an in-vitro investigation of the interaction of progesterone on isolated follicles at different stages of development will be important in aiding understanding of these complex interactions.

We thank H. M. Fraser for kindly measuring serum progesterone and the Medical Research Council for a grant to R. G. Gosden.

\section{References}

Baird, D.T., Backstrom, T., McNeilly, A.S., Smith, S.K. \& Wathen, C.G. (1984) Effect of enucleation of the corpus luteum at different stages of the luteal phase of the human menstrual cycle on subsequent follicular development. J. Reprod. Fert. 70, 615-624.

Buffier, G. \& Roser, S. (1974) New data concerning the role played by progesterone in the control of follicular growth in the rat. Acta Endocr. 75, 569-578.

Faddy, M.J., Jones, E.C. \& Edwards, R.G. (1976) An analytical model for ovarian follicle dynamics. J. exp. Zool. 197, 173-186.

Faddy, M.J. \& Jones, M.C. (1988) Fitting time dependent multi-compartmental models: a case study. Biometrics 44, 587-593.

Faddy, M.J., Telfer, E. \& Gosden, R.G. (1987) The kinetics of preantral follicle development in ovaries of CBA/Ca mice during the first 14 weeks of life. Cell \& Tiss. Kinet. 20, 551-560.

Faddy, M.J., Gosden, R.G. \& Edwards, R.G. (1983) Ovarian follicle dynamics in mice: a comparative study of three inbred strains and an $\mathrm{F}_{1}$ hybrid. $J$. Endocr. 96, 23-33.

Fraser, H.M. (1983) LHRH agonist in stump tailed monkeys. Endocrinology 112, 245-253.

Goodman, A.L. \& Hodgen, G.D. (1977) Systematic versus intraovarian progesterone replacement after luteectomy in rhesus monkeys; differential patterns of gonadotropins and follicular growth. J. Clin. Endocr. Metab. 45, 837-840.

Greenwald, G.S. (1966) Ovarian follicular development and pituitary FSH and $\mathrm{LH}$ content in the pregnant rat. Endocrinology 79, 572-578.

Greenwald, G.S. (1977) Exogenous progesterone: influence on ovulation and hormone levels in the cyclic hamster. J. Endocr. 7, 151-155.

Greenwald, G.S. \& Choudary, J.B. (1969) Follicular development and induction of ovulation in the pregnant mouse. Endocrinology 84, 1512-1516.

Halpin, D.M.G., Charlton, H.M. \& Faddy, M.J. (1986) Effects of gonadotrophin deficiency on follicular development in hypogonadal $(\mathrm{hpg})$ mice. J. Reprod. Fert. 78, 119-125.

Jeffcoate, S.L. (1986) Anterior pituitary hormones. In Scientific Foundations of Obstetrics \& Gynaecology, pp. 443-448. Eds E. Philipp, J. Barnes \& M. Newton. Heinemann, London.

Kent, J. (1973) Some effects of steroids on the immature mouse ovary in vitro. J. Reprod. Fert. 34, 297-303.
Linkie, D.M. \& Niswender, G.D. (1972) Serum levels of prolactin, luteinizing hormone and follicle stimulating hormone during pregnancy in the rat. Endocrinology 90, 632-637.

McCormack, J.T. \& Greenwald, G.S. (1974) Progesterone and oestradiol-17 7 concentrations in peripheral plasma during pregnancy in the mouse. J. Endocr. 62, 101-107.

Mandl, A.M. \& Zuckerman, S. (1951) Numbers of normal and atretic oocytes in unilaterally spayed rats. J. Endocr. 7, 112-119.

Moore, P.J. \& Greenwald, G.S. (1974) Effect of hypophysectomy and gonadotropin treatment on follicular development and ovulation in the hamster. Am. J. Anat. 139, 37. 48.

Murr, S.M., Stabenfeldt, G.H., Bradford, G.E. \& Geschwind, I.I. (1974) Plasma progesterone during pregnancy in the mouse. Endocrinology 94, 1209-1211.

Numazawa, A. \& Kawashima, S. (1982) Morphometric studies on ovarian follicles and corpora lutea during the oestrous cycle in the mouse. J. Reprod. Fert. 64, 275-283.

Osman, P. (1985) Rate and course of atresia during follicular development in the adult cyclic rat. $J$. Reprod. Fert. 73, 261-270.

Osman, P. (1986) Morphometric analysis of follicular dynamics in pregnant and pseudopregnant rats. $J$. Reprod. Fert. 76, 11-22.

Pedersen, T. \& Peters, H. (1971) Follicle growth and cell dynamics in the mouse ovary during pregnancy. Fert. Steril. 22, 42-52.

Richards, J.S. (1980) Maturation of ovarian follicles: actions and interactions of pituitary and ovarian hormones on follicular cell differentiation. Physiol. Rev. 60, 51-89.

Rabson, J.M. (1938) Quantitative data on the inhibition of oestrus by testosterone, progesterone, and certain other compounds. J. Physiol. 92, 371-382.

Schreiber, J.R., Nakamura, K. \& Erickson, G.F. (1980) Progestins inhibit FSH steroidogenesis in cultured rat granulosa cells. Mol. Cell. Endocr. 19, 165-173.

Setty, S.L. \& Mills, T.M. (1987) The effects of progesterone on follicular growth in the rabbit ovary. Biol. Reprod. 36, 1247-1252.

Received 8 July 1991 